S. Kotsios, I. Kostarakos

\title{
CONTROLLING NATIONAL INCOME AND PUBLIC DEBT VIA FISCAL POLICY. A MODEL MATCHING ALGORITHMIC APPROACH
}

National and Kapodistrian University of Athens, 30, ul. Panepistimiu, Athens, 10559, Greece

Our aim in this paper is to present an application of the exact model matching approach to a linear, discrete macroeconomic model with delays. The solution is based on algebraic methods and the development, in computer algebra software, of appropriate symbolic algorithms that produce a class of feedback laws as a solution. These feedback laws constitute a class of potential policies that the policymaker can implement on the system and modify its dynamics so that predetermined, desired targets are met. In order to see the effects of different policies, we implement two feedback laws on the system at hand. Refs 9. Table 1.

Keywords: algebraic methods, linear discrete systems, model matching, symbolic algorithms.

\author{
С. Котсиос, И. Костаракос
}

\section{УПРАВЛЕНИЕ НАЦИОНАЛЬНЫМ ДОХОДОМ И ГОСУДАРСТВЕННЫМ ДОЛГОМ ПОСРЕДСТВОМ ФИСКАЛЬНОЙ ПОЛИТИКИ. МОДЕЛЬ, ПОДСТРАИВАЮЩАЯ АЛГОРИТМ}

\begin{abstract}
Афинский национальный университет имени Каподистрии, Греция, 10559, Афины, ул. Панепистимиу, 30

Целью в данной работе является применение модели, подстраивающейся к линейной, дискретной макроэкономической модели с задержками. Решение основано на алгебраических методах и на развитии алгебраического программного обеспечения соответствующих символьных алгоритмов, которые создают класс законов обратной связи. Эти законы обратной связи представляют собой класс потенциальных стратегий, которые политик может реализовать, управляя системой и изменением ее динамики так, чтобы достигались заранее заданные цели. Для того чтобы продемонстрировать эффект использования различных стратегий, мы реализуем два закона обратной связи в рассматриваемой системе. Библиогр. 9 назв. Табл. 1.

Ключевые слова: алгебраическиу методы, линейные дискретные системы, модель, подстраивающая алгоритм.
\end{abstract}

The scope of this paper is to examine the problem of shaping the time path of a linear, discrete macroeconomic model with delays, using appropriate feedback controllers so that its outputs exactly follow a predetermined trajectory of desired values. This is, essentially, a tracking problem that can be tackled utilizing the (exact) model matching approach under which we want to modify the nominal plant in such a way that it matches a desired system. In particular, it amounts to calculating appropriate linear, causal feedback laws such that the resulting closed-loop system is identical to a desired one that produces the requested output values. Although the linear model matching approach has been extensively studied and is well documented in the control literature (see, among others [1-4]), it has not, to the

Kotsios Stelios - doctor of philosophical sciences, professor; :skotsios@econ.uoa.gr

Kostarakos Ilias - master; hkostarak@econ.uoa.gr

Котсиос Стелеос - доктор философских наук, профессор; :skotsios@econ.uoa.gr

Костаракос Илиас - магистр; hkostarak@econ.uoa.gr

(c) Санкт-Петербургский государственный университет, 2016 
extent of our knowledge, been used in the economic policy literature (for some exceptions, see $[5,6]$ ) and for a nonlinear application, (see [7, 8]) in spite of its advantages. The main advantage of this approach is that it relies only on the use of algebraic tools. Moreover, the algorithmic nature of the solution allows for the calculation of a class of feedback laws appropriate for solving the model matching problem. From an economic policy point of view, this implies that the policymaker has at his disposal a set of policy rules available that can be used, depending on the particular problem at hand.

The macroeconomic model. We consider a linear deterministic macroeconomical model, described by the following difference equations:

$$
\begin{gathered}
Y(t)=C(t)+I(t)+\lambda_{0} G(t)+\lambda_{1} G(t-1)+\lambda_{2} G(t-2), \\
C(t)=(1-s) Y^{d}(t-1)+s Y^{d}(t-2), \\
Y^{d}(t)=Y(t)-T(t), \\
T(t)=\tau Y(t-1)+E(t), \\
I(t)=\nu(Y(t-1)-Y(t-2)), \\
B(t)=(1+r) B(t-1)+G(t)-T(t),
\end{gathered}
$$

where $Y(t)$ denotes the national income (GDP); $C(t)$ is consumption; $I(t)$ is investment; $B(t)$ is the stock of public debt; $G(t)$ stands for government expenditures; $Y^{d}(t)$ is the disposal income; $T(t)$ the tax receipts and $E(t)$ is a distinct form of taxation, assumed to represent extra tax on wealth. The $\lambda_{i}$ parameters indicate that the decision regarding government expenditures in period $t$ affects the level of GDP in period $t+i$; that is, there is a delay in the realization of the effects of changes in government expenditures on GDP levels. Equations (1)-(5) constitute a variant of the standard multiplier-accelerator model introduced in [9] whereas equation (6) is the government budget constraint. Regarding the parameters of the system, $s \in(0,1)$ is the marginal propensity to save, $\tau \in(0,1)$ is the (constant) tax rate, $\nu>0$ is the accelerator and $r \in(0,1)$ is the constant interest on previously issued public debt. Apart from the $\lambda_{i}$ parameters, delays are also introduced into the model through equations (2) and (5), since it is assumed that the current levels of $C(t), I(t)$, depend upon changes in lagged values of $Y(t)$.

After all the necessary substitutions and some algebra we end up with the discrete system

$$
\begin{gathered}
Y(t)-a_{1} Y(t-1)-a_{2} Y(t-2)+a_{3} Y(t-3)=\lambda_{0} G(t)+\lambda_{1} G(t-1)+ \\
+\lambda_{2} G(t-2)-a_{4} E(t-1)-a_{5} E(t-2), \\
B(t)-(1+r) B(t-1)-\tau Y(t-1)=G(t)-E(t),
\end{gathered}
$$

here $a_{1}=1+\nu-s, a_{2}=s-\nu-\tau(1-s), a_{3}=s \tau, a_{4}=1-s, a_{5}=s$. This is a discrete input-output control system, with $G(t)$ and $E(t)$ being the inputs, and $Y(t)$ and $B(t)$ the outputs. Certain initial conditions are assigned to the system, thus permitting the calculation of all future values of the outputs for any given input sequences.

Using the notion of the $q$-operator, that is, a lag operator defined as: $q^{m} f(t)=$ $f(t-m)$, for any sequence $f(t), t=0,1,2, \ldots$, the system can be expressed in the following so-called algebraic form:

$$
\begin{gathered}
{\left[\begin{array}{cc}
1-a_{1} q-a_{2} q^{2}-a_{3} q^{3} & 0 \\
-\tau q & 1-(1+r) q
\end{array}\right]\left[\begin{array}{c}
Y(t) \\
B(t)
\end{array}\right]=\left[\begin{array}{cc}
\lambda_{0}+\lambda_{1} q+\lambda_{2} q^{2} & a_{4} q+a_{5} q^{2} \\
1 & -1
\end{array}\right]\left[\begin{array}{c}
G(t) \\
E(t)
\end{array}\right]} \\
\Longleftrightarrow \quad \mathcal{D}(q) \mathbf{z}(t)=\mathcal{K}(q) \mathbf{u}(t),
\end{gathered}
$$


where $\mathbf{z}(t)=(Y(t), B(t))^{T}, \mathbf{u}(t)=(G(t), E(t))^{T}(T$ denotes transpose $)$ and $\mathcal{D}, \mathcal{K}$ are the corresponding $q$-polynomial matrices.

The model matching problem for the macroeconomic model. The economic policy problem that we aim to solve is that of designing appropriate linear, causal feedback laws such that the resulting closed-loop system exactly tracks the desired sequence of outputs. In order to solve the problem at hand we follow the exact model matching approach, which describe in brief. First, the desired values of the outputs, the so-called reference sequences (say, $Y^{*}(t)$ and $B^{*}(t)$ respectively) are determined by the policymaker. Then, a linear system is constructed, that produces as outputs these reference sequences. Such a linear system can be represented as

$$
\mathcal{D}^{d}(q) \mathbf{z}^{*}(t)=\mathcal{K}^{d}(q) \mathbf{u}_{c}(t),
$$

here $\mathbf{z}^{*}(t)=\left(Y(t)^{*}, B(t)^{*}\right)^{T}$ and $\mathcal{D}^{d}(q), \mathcal{K}^{d}(q)$, are appropriate $q$-polynomial matrices and $\mathbf{u}_{c}(t)$ is the command input. System (8) is known as the desired system. Now, the problem upon discussion is that of finding linear, causal feedback laws of the form

$$
\mathcal{R}(q) \mathbf{u}(t)=\mathcal{T}(q) \mathbf{u}_{c}(t)-\mathcal{S}(q) \mathbf{z}(t),
$$

where $\mathcal{R}(q), \mathcal{T}(q), \mathcal{S}(q)$, are $q$-polynomial matrices to be determined, such that the resulting closed-loop system will be identical (i. e. matched) to the desired system (8). The following theorems describe the solution method and characterize the solution set.

Theorem 1. Let us suppose that a feedback law of the form (9) is connected to the nominal plant (7). The obtained closed-loop system will give the same output with that of the desired system (8) under the same initial conditions and the same input, if and only if the next relations hold:

$$
\begin{gathered}
\mathcal{R}(q) \mathcal{D}(q)+\mathcal{K}(q) \mathcal{S}(q)=\mathcal{D}^{d}(q), \\
\mathcal{K}(q) \mathcal{T}(q)=\mathcal{K}^{d}(q), \\
\mathcal{R}(q) \mathcal{K}(q)=\mathcal{K}(q) \mathcal{R}(q), \\
\mathcal{R}=I+\overline{\mathcal{R}}, \quad \mathcal{D}=I+\overline{\mathcal{D}}
\end{gathered}
$$

( $I$ is the identity matrix).

P r o o f. For the sake of presentation we drop the $q$ symbol. Let us suppose now, that $\mathbf{z}(t)=\mathbf{z}^{*}(t), t=0,1, \ldots, m-1$, where $m$ is the largest delay, appeared in the equations of the system. In other words, we have identical initial conditions. We shall prove that $\mathbf{z}(m)=\mathbf{z}^{*}(m)$.

For $t=m$ we have: $\mathcal{D} \mathbf{z}(m)=\mathcal{K} \mathbf{u}(m) \Rightarrow(I+\overline{\mathcal{D}}) \mathbf{z}(m)=\mathcal{K} \mathbf{u}(m) \Rightarrow \mathbf{z}(m)=\mathcal{K} \mathbf{u}(m)-$ $\overline{\mathcal{D}} \mathbf{z}(m)$. But $\mathcal{R} \mathbf{u}(t)=\mathcal{T} \mathbf{u}_{c}(t)-\mathcal{S} \mathbf{z}(t)$ and $\mathcal{R}=I+\overline{\mathcal{R}} \Rightarrow \mathbf{u}(t)=\mathcal{T} \mathbf{u}_{c}(t)-\mathcal{S} \mathbf{z}(t)-\overline{\mathcal{R}} \mathbf{u}(t)$. By setting $t=m$ and substitution we get

$$
\mathbf{z}(m)=\mathcal{K} \mathcal{T} \mathbf{u}_{c}(m)-\mathcal{K} \mathcal{S} \mathbf{z}(m)-\mathcal{K} \overline{\mathcal{R}} \mathbf{u}(m)-\overline{\mathcal{D}} \mathbf{z}(m)
$$

All the quantities at the right hand side of (14) are delays of the sequences and thus we can substitute the sequence $\mathbf{z}(t)$ with $\mathbf{z}^{*}(t)$, since $\mathbf{z}(t)=\mathbf{z}^{*}(t)$, for $t=0,1,2, \ldots, m-1$. From equations (12) and (13) we get $(I+\overline{\mathcal{R}}) \mathcal{K}=\mathcal{K}(I+\overline{\mathcal{R}})$ which implies: $\overline{\mathcal{R}} \mathcal{K}=\mathcal{K} \overline{\mathcal{R}}$. From equations $(10)$ and $(13)$ we get $(I+\overline{\mathcal{R}})(I+\overline{\mathcal{D}})+\mathcal{K} \mathcal{S}=\mathcal{D}^{d}$ or $\mathcal{K} \mathcal{S}=\mathcal{D}^{d}-I-\overline{\mathcal{R}}-\overline{\mathcal{D}}-\overline{\mathcal{R} \mathcal{D}}$. Substituting all the above into (14) we have 


$$
\begin{aligned}
& \mathbf{z}(m)=\mathcal{K} \mathcal{T} \mathbf{u}_{c}(m)-\mathcal{D}^{d} \mathbf{z}^{*}(m)+\mathbf{z}^{*}(m)+\overline{\mathcal{R}} \mathbf{z}^{*}(m)+ \\
& +\overline{\mathcal{D}} \mathbf{z}^{*}(m)+\overline{\mathcal{R} D} \mathbf{z}^{*}(m)-\mathcal{K} \overline{\mathcal{R}} \mathbf{u}(m)-\overline{\mathcal{D}} \mathbf{z}^{*}(m) .
\end{aligned}
$$

Using the facts that $\mathcal{K} \mathcal{T}=\mathcal{K}^{d}, \mathcal{D}^{d} \mathbf{z}^{*}(m)=\mathcal{K}^{d} \mathbf{u}_{c}(m), \overline{\mathcal{R}} \mathcal{D} \mathbf{z}^{*}(m)=\overline{\mathcal{R}}(I+\overline{\mathcal{D}}) \mathbf{z}^{*}(m)=$ $\overline{\mathcal{R}} \mathcal{K} \mathbf{u}(m)=\mathcal{K} \overline{\mathcal{R}} \mathbf{u}(m)$ the latter becomes $\mathbf{z}(m)=\mathbf{z}^{*}(m)$ as requested. By induction we get the final result $\mathbf{z}(t)=\mathbf{z}^{*}(t)$, for any $t$, and the theorem has been proved.

Theorem 2. Let us suppose that the polynomial matrices $\mathcal{R}_{0}, \mathcal{S}_{0}, \mathcal{T}$, solve the system (10)-(12), then the polynomial matrices $\mathcal{R}=\mathcal{R}_{0}+\mathcal{Q K}, \mathcal{S}=\mathcal{S}_{0}-\mathcal{Q D}, \mathcal{T}$ are solutions too, for any arbitrary polynomial matrix $\mathcal{Q}$ such that $\mathcal{Q K}=\mathcal{K} \mathcal{Q}$

P r o o f. Substituting in $(10)$, we get $\left(\mathcal{R}_{0}+\mathcal{Q K}\right) \mathcal{D}+\mathcal{K}\left(\mathcal{S}_{0}-\mathcal{Q D}\right)=\mathcal{R}_{0} \mathcal{D}+\mathcal{Q K} \mathcal{D}+$ $\mathcal{K} \mathcal{S}_{0}-\mathcal{K} \mathcal{Q D}$. Using the facts that $\mathcal{R}_{0}$ and $\mathcal{S}_{0}$ are solutions of the system and that $\mathcal{Q K}=\mathcal{K} \mathcal{Q}$, we conclude that the above quantity is equal to $\mathcal{D}^{d}$. Furthermore, we have $\left(\mathcal{R}_{0}+\mathcal{Q K}\right) \mathcal{K}=\mathcal{K}\left(\mathcal{R}_{0}+\mathcal{Q K}\right) \Leftrightarrow \mathcal{R}_{0} \mathcal{K}+\mathcal{Q K} \mathcal{K}=\mathcal{K} \mathcal{R}_{0}+\mathcal{K} \mathcal{Q} \mathcal{K}$. Using again the facts that $\mathcal{Q K}=\mathcal{K} \mathcal{Q}$ and $\mathcal{R}_{0} \mathcal{K}=\mathcal{K} \mathcal{R}_{0}$ we conclude that the above relation is valid. From all the above we get that the new matrices are solutions of the system.

The algorithmic solution. Now, in order to solve the problem of designing appropriate polynomial matrices $\mathcal{R}, \mathcal{S}$ and $\mathcal{T}$ we developed two symbolic algorithmic procedures, the "Desired System" algorithm and the "Income and Debt Matching" algorithm. The "Desired System" algorithm is used for the calculation of the polynomial matrices $\mathcal{D}^{d}, \mathcal{K}^{d}$, while the "Income and Debt Matching" algorithm produces the polynomial matrices $\mathcal{R}, \mathcal{S}$ and $\mathcal{T}$ of the requested feedback law.

\section{The "Desired System" algorithm}

Input: The degrees $n$ and $m$ of the output and input polynomials. The desired sequences: $y^{*}(t), \mathbf{u}^{*}(t), t=0, \ldots, k$.

Output: The numbers $a_{i}, i=0, \ldots, n, b_{j}, j=0, \ldots, m$.

Step 1. We construct the system of algebraic equations for $t=0,1, \ldots, k$ :

$$
a_{0} y^{*}(t)+a_{1} y^{*}(t-1)+\cdots+a_{n} y^{*}(t-n)=b_{0} \mathbf{u}^{*}(t)+\cdots+b_{m} \mathbf{u}^{*}(t-m) .
$$

Step 2. We solve the above system with respect to the unknown coefficients $a_{i}$, $i=0, \ldots, n, b_{j}, j=0, \ldots, m$.

\section{The "Income and Debt Matching" algorithm}

Input: The parameters $\nu, s, \tau, r$ and $\lambda_{0}, \lambda_{1}, \lambda_{2}$. The initial conditions: $Y(0), B(0), G(0)$, $E(0), Y(3), B(3), G(3), E(3)$. The input: $\mathbf{u}_{c}(t)$. The reference sequences: $Y^{*}(t), B^{*}(t)$.

Output: The polynomial matrices: $\mathcal{R}\left(\theta^{*}\right), \mathcal{S}\left(\theta^{*}\right), \mathcal{T}\left(\theta^{*}\right)$.

Step 1. Form the polynomial matrices $\mathcal{D}$ and $\mathcal{K}$.

Step 2. Using the "Desired System" algorithm, construct a family of desired polynomial matrices $\mathcal{D}^{d}(\theta), \mathcal{K}^{d}(\theta)$ such that the output of the system: $\mathcal{D}^{d}(\theta) \mathbf{z}^{*}(t)=$ $\mathcal{K}^{d}(\theta) \mathbf{u}_{c}(t)$ is exactly equal to the reference sequences. Note: the vector of the parameters $\theta$ consists of the parameters $a_{i}, b_{j}$ used in the construction of the desired system.

Step 3. Construct the system of polynomial equations of Theorem 1.

Step 4. Construct the coefficient matrix of the above system $\mathcal{A}$ and the corresponding augmented matrix $\mathcal{A}^{\text {aug }}(\theta)$. 
Step 5. Find a parameter $\theta^{*} \operatorname{such}$ that $\operatorname{rank}(\mathcal{A})=\operatorname{rank}\left(\mathcal{A}^{\operatorname{aug}}\left(\theta^{*}\right)\right)$ and $R_{\theta^{*}}=I+\bar{R}_{\theta^{*}}$.

Step 6. Solve the system of equations of Theorem 1 , with respect to the quantities $\mathcal{R}\left(\theta^{*}\right), \mathcal{S}\left(\theta^{*}\right), \mathcal{T}\left(\theta^{*}\right)$, for this particular $\theta^{*}$ value.

It is a straightforward result of Theorem 2, that the output of the "Income and Debt Matching" Algorithm, solves the national income-debt tracking problem upon discussion.

An application of the algorithm. In order to obtain a better understanding of the effects of the proposed methodology on the macroeconomic system under consideration, we run some simulations for a model economy. In particular, let us suppose that $s=0.2$, $\tau=0.4, \nu=1, r=5 \%, \lambda_{0}=0.4, \lambda_{1}=0.3, \lambda_{2}=0.3$, so that the open-loop system becomes:

$$
\begin{gathered}
Y(t)=1.8 Y(t-1)-1.12 Y(t-2)-0.08 Y(t-3)+0.4 G(t)+ \\
+0.3 G(t-1)+0.3 G(t-2)-0.8 E(t-1)-0.8 E(t-2), \\
B(t)=1.05 B(t-1)-0.4 Y(t-1)+G(t)-E(t)
\end{gathered}
$$

and the initial conditions are $Y(3)=100, B(3)=215$. For the purposes of our simulations, we assume that the policymaker aims for $1 \%$ growth in the levels of $Y$ and $1 \%$ decrease in the levels of $B$, per period. Then, by applying the "Desired System" algorithm and the "Income and Debt Matching" algorithm we obtain a class of linear, causal feedback laws as a solution. We present the time paths for the inputs, resulting from two of the (infinitely many) feedback laws (see Table):

\section{Values of the control variables under two feedback laws}

\begin{tabular}{|c|c|c|c|c|}
\hline$t$ & $G_{1}(t)$ & $E_{1}(t)$ & $G_{2}(t)$ & $E_{2}(t)$ \\
\hline 4 & 31.54 & 4.44 & 35.5 & 8.45 \\
5 & 30.54 & 2.91 & 40.3 & 12.68 \\
6 & 32.92 & 4.76 & 49.5 & 21.3 \\
7 & 33.19 & 4.49 & 50.4 & 58.7 \\
\hline
\end{tabular}

The $G_{1}(t), E_{1}(t)$ values correspond to the following feedback law:

$$
\begin{gathered}
G_{1}(t)=1.44 G 1(t-1)-0.366 G 1(t-2)+1.36 Y(t-1)-2.48 Y(t-2)- \\
-0.645 Y(t-3)+2.27 B(t-1)-1.66 B(t-2)+0.234 B(t-3), \\
E_{1}(t)=1.44 E 1(t-1)-0.366 E 1(t-2)+1.07 Y(t-1)-1.707 Y(t-2)- \\
-1.113 Y(t-3)+4.049 B(t-1)-3.53 B(t-2)+0.35 B(t-3),
\end{gathered}
$$

whereas the $G_{2}(t), E_{2}(t)$ values correspond to the following feedback law:

$$
\begin{gathered}
G_{2}(t)=1.44 G 2(t-1)-0.366 G 2(t-2)+1.33 Y(t-1)-2.45 Y(t-2)- \\
-0.642 Y(t-3)+2.26 B(t-1)-1.63 B(t-2)+0.236 B(t-3), \\
E_{2}(t)=1.44 E 2(t-1)-0.366 E 2(t-2)+1.05 Y(t-1)-1.68 Y(t-2)- \\
\quad-1.11 Y(t-3)+4.046 B(t-1)-3.51 B(t-2)+0.354 B(t-3) .
\end{gathered}
$$

As we can see from the table, under feedback law (16) the values of the inputs necessary for simultaneously reaching the reference sequences are much higher compared 
to the values under feedback law (15). This implies that, since the size of the policy interventions under feedback law (15) are essentially politically infeasible, the policymaker should choose the feedback law provides a smoother transition path for the inputs.

Concluding remark. Our aim in this paper was to present an application of the model matching approach for the design of economic policy, i. e. for the purpose of shaping the time path of a macroeconomic model. From a control theory perspective, the advantages of this approach are that it is based on algebraic tools and that is provides a general class of feedback laws as a solution. The algorithmic nature of the solution ensures that this approach can readily be extended to macroeconomic models with more delays than those considered in this paper.

\section{References}

1. Astrom K. J., Wittenmark B. Computer-Controlled Systems: Theory and Design. 3rd ed., New York, Dover Publ., 2011, 576 p.

2. Goodwin G., Sin K.S. Adaptive filtering prediction and control. New York, Dover Publ., 2009, $560 \mathrm{p}$.

3. Kucera V. Exact model matching, polynomial equation approach. Intern. Journal of systems science, 1981, vol. 12, no. 12, pp. 1477-1484.

4. Kucera V., Sebek M. Exact model matching of discrete linear systems. Systems and Control Letters, 1982, vol. 1, no. 5, pp. 321-325.

5. Kostarakos I., Kotsios S. Fiscal policy design in Greece in the aftermath of the crisis: an algorithmic approach. Computation Economics, 2016 (unpublished).

6. Kostarakos I., Kotsios S. Feedback policy rules for goverment spending: an algorithmic approach. Of Economic structures, 2016 (unpublished).

7. Kotsios S., Leventidis J. A feedback policy for a modified Samuelson-Hicks model. Intern. Journal of systems science, 2004, vol. 35, no. 6, pp. 331-341.

8. Prasolov A. V. Some quantitative methods and models in economic theory. New York, Nova Science Publ., 2016, 301 p.

9. Samuelson P. Interactions between the multiplier analysis and the principle of acceleration. Review of Economics and Statistics, 1939, vol. 21, no. 2, pp. 75-78.

For citation: Kotsios S., Kostarakos I. Controlling national income and public debt via fiscal policy. A model matching algorithmic approach. Vestnik of Saint Petersburg University. Series 10. Applied mathematics. Computer science. Control processes, 2016, issue 4, pp. 86-91. DOI: $10.21638 / 11701 / \mathrm{spbu} 10.2016 .408$

Статья рекомендована к печати проф. Л. А. Петросяном.

Статья поступила в редакцию 22 июля 2016 г.

Статья принята к печати 29 сентября 2016 г. 